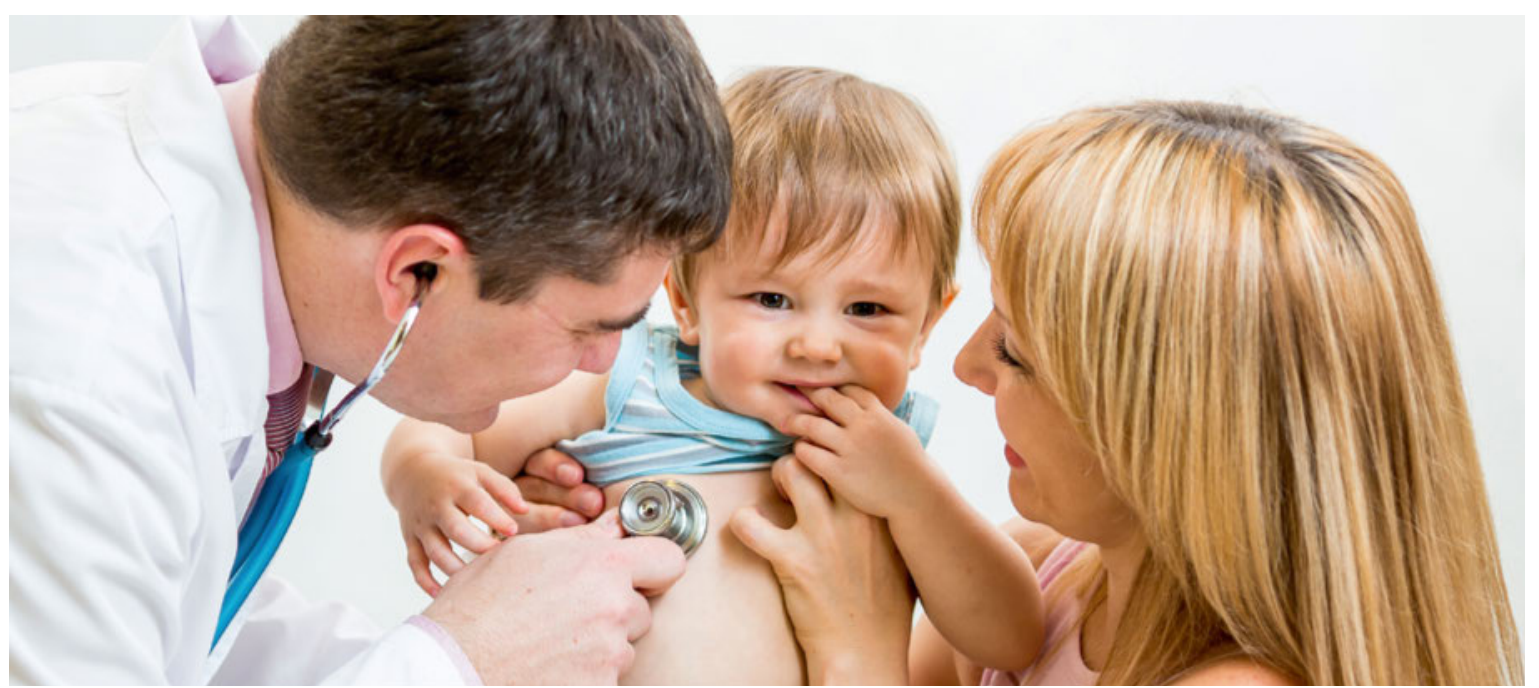

\title{
Die Kinder- und Jugendmedizin von morgen - ein Ausblick
}

\author{
Barbara Wildhaber ${ }^{a}$, Hélène Beutler ${ }^{b}$, Urs Frey $^{c}$, Nicole Pellaud ${ }^{d}$, Michaël Hofer ${ }^{\mathrm{e}}$ \\ a Schweizerische Gesellschaft für Kinderchirurgie (SGKC), Genf-Lausanne; ${ }^{b}$ Schweizerische Gesellschaft für Kinder- und Jugendpsychiatrie und \\ -psychotherapie (SGKJPP), Neuenburg; ' Kollegium der Chefärzte der Kinderkliniken A, Basel; ' Schweizerische Gesellschaft für Pädiatrie (SGP), Genf; \\ e Ärztliche Union für Kinder und Jugendliche (fPmh: foederatio Paedo medicorum helveticorum), Lausanne-Genf
}

\section{Die Gesundheit des Kindes und sein Umfeld}

Jedes Kind hat Anrecht auf das erreichbare Höchstmass an Gesundheit sowie auf Inanspruchnahme von Einrichtungen zur Behandlung von Krankheiten und zur Wiederherstellung der Gesundheit (internationales Übereinkommen über die Rechte des Kindes, Artikel 24). Wenn es mit einer Krankheit konfrontiert ist, «darf das Kind Angst haben, weinen und getröstet werden. Das kranke Kind hat seinem Zustand entsprechend Anrecht auf die bestmögliche Gesundheitsversorgung. Das Kind, seine Eltern oder deren Vertreter, haben Anrecht auf eine korrekte und angepasste Information zur Krankheit des Kindes und den zu verordnenden Behandlungen. Das kranke Kind, welches über die körperlichen und psychologischen Möglichkeiten verfügt, soll weiterhin an schulischen, spielerischen, kulturellen oder sportlichen Aktivitäten teilnehmen, muss flexible Bindungsstrukturen zwischen Familie, Schule und Jugendbewegungen geniessen können.» Diese Sätze entstammen der "Charta für Kinder im Krankenhaus» (EACH: www.each-for-sick-children.org), einer europäischen Verbandsinitiative, welche 1988 in Leiden durch verschiedene europäische Verbände anlässlich der ersten europäischen Konferenz «Kind im Krankenhaus» verfasst und seither von den meisten
Einrichtungen, die Kinder betreuen, übernommen wurde.

Integraler Bestandteil der pädiatrischen Versorgung bei allen gesundheitlichen Problemen ist die Sorge für eine gute Entwicklung der Kinder, gemeinsam mit allen betroffenen Partnern; Familien und Fachpersonen.

Nichtsdestotrotz sehen wir uns vor grossen Herausforderungen, vor allem was die Forschung und deren Finanzierung wie auch die Gesamtbehandlung des Kindes betrifft

\section{Die Kinder- und Jugendmedizin im Fokus der Forschung}

Kinder und Jugendliche haben das Recht, von den medizinischen Fortschritten und damit von einer qualitativ hochstehenden Kinder- und Jugendmedizin zu profitieren.

In Folge der unzureichend entwickelten Forschung in der Kinder- und Jugendmedizin ist allerdings der $\mathrm{Zu}$ gang zu Medikamenten für Kinder und Jugendliche viel eingeschränkter, als dies bei Erwachsenen der Fall ist. Im Weiteren stellen sich für die Forschung in der Pädiatrie hohe ethische und sicherheitstechnische Bedingungen. Mit der Gründung von SwissPedNet steht den Forschern ein professionelles Leistungsangebot zur Verfügung. Nutzung und Entwicklung dieser Plattform müssen unbedingt gefördert werden. 


\section{Die Kinder- und Jugendmedizin und ihre Finanzierung}

Die Entwicklung der letzten Jahre deutet darauf hin, dass die Finanzierung der Gesundheitsversorgung von Kindern und Jugendlichen in Zukunft nicht sichergestellt ist. Die Vielzahl von seltenen und komplexen Krankheiten bedingt grosse Investitionen an Zeit, Energie und Zusammenarbeit. Diesen Aspekten wird durch die Tarifsysteme (TARMED, DRG) zu wenig Rechnung getragen; eine Reform, basierend auf einer ganzheitlichen und detaillierten Analyse ist erforderlich.

\section{Die fPmh}

2006 haben sich die schweizerischen medizinischen Fachgesellschaften für Kinder und Jugendliche - die Schweizerische Gesellschaft für Pädiatrie (SGP), die Schweizerische Gesellschaft für Kinder- und Jugendpsychiatrie und -Psychotherapie (SGKJPP) und die Schweizerische Gesellschaft für Kinderchirurgie (SGKC) - zusammengesetzt, um die foederatio Paedo medicorum helveticorum (fPmh), oder Ärztliche Union für Kinder und Jugendliche, zu gründen. Dieser Dachverband setzt sich für die medizinische Betreuung der pädiatrischen Bevölkerungsgruppe ein. Das Hauptziel ist die Sicherstellung einer medizinisch angemessenen Versorgung, die auf die Bedürfnisse von Kindern und Jugendlichen zugeschnitten ist.

Wir wollen weiterhin die Versorgungsqualität sicherstellen, ungeachtet der in unseren Institutionen verbreiteten Gesetze der Wirtschaftlichkeit.

Politiker und das medizinische Fachpersonal sehen sich vor die ethische Herausforderung gestellt, das Gleichgewicht zwischen hochstehender Versorgungsqualität und der ausgewogenen Ressourcenverteilung zu fördern und zu wahren.

Wir müssen die pädiatrische Versorgung individuell und gemeinschaftlich gestalten, die adaptierte Einzelbetreuung des Kindes dabei sicherstellen, und das, indem den Standards Rechnung getragen wird.

Diese doppelte Einschränkung ist der Kern der Herausforderung, welcher sich die heutige Medizin stellen muss, wie dies auch die Schweizerische Akademie der Medizinischen Wissenschaften (SAMW) in den letzten Veröffentlichungen gezeigt hat (http://www.samw.ch/ de/Aktuell/News.html).

Die adäquate Versorgung von Kindern und Jugendlichen steht auf dem Spiel: Sie muss trotz ökonomischen Bedenken weiterhin sichergestellt werden.

\section{Unumgängliche Folgerungen}

Wir müssen uns für eine vermehrte und zielgerichtete Analyse der Gesamtsituation der Versorgung und Betreuung der Kinder und Jugendlichen mit gesundheitlichen Problemen einsetzen. Wir müssen uns dabei auf die Prophylaxe und die Früherkennung der Erkrankungen der Kinder und Jugendlichen konzentrieren, insbesondere die normale Entwicklung unterstützen und favorisieren, um somit chronische Krankheiten im Erwachsenenalter zu verhindern.

\section{Die fPmh setzt sich ein für:}

1. den klinischen Fortschritt, die Nachwuchsförderung und die Forschung für eine gesamtheitliche Gesundheitsbetreuung der Kinder und Jugendlichen, welche deren Entwicklung und Umfeld einbezieht;

2. eine langfristige ökonomische und soziologische Analyse der aktuellen Situation der Kinder- und Jugendmedizin (Umfragen innerhalb der Schweizer Bevölkerung, Kartographie der Schweizer Bedürfnisse);

3. eine Analyse der Umweltveränderungen (psychosozial, ernährungstechnisch, physikalisch-chemisch, multikulturell) und ihres Einflusses auf die langfristige Gesundheit der Kinder und Jugendlichen;

4. eine Analyse der Interaktion zwischen Kinder- und Erwachsenenmedizin («Transition»);

5. die Förderung der Forschung, mit hohen Qualitätsanforderungen auf wissenschaftlicher und ethischer Ebene (SwissPedNet: www.swisspednet.ch);

6. die Information der Öffentlichkeit, für den Erhalt der notwendigen Mittel zugunsten der Kinder- und Jugendmedizin, damit eine optimale Versorgung der Kinder und Jugendlichen auch in Zukunft sichergestellt werden kann.

Aus dieser Analyse wird eine Definition und Ausrichtung der Kinder- und Jugendmedizin im Hinblick auf die Sicherstellung der Versorgungsqualität auch in Zukunft abgeleitet werden können. So wird es auch möglich sein, eine Vision der Kinder- und Jugendmedizin zu entwickeln.

Eines Tages werden unsere Kinder Erwachsene und Mitbürger sein: Sie werden dann die Gesellschaftssolidarität verantworten. Die Dauerhaftigkeit der Investitionen in die Gesundheit der Kinder und Jugendlichen liegt auf der Hand und wird zu einer besseren Kostenkontrolle in der Medizin der Zukunft führen.

Bildnachweis

(c) Oksun70 | Dreamstime.com 